\title{
CARACTERÍSTICAS PSICOMÉTRICAS PRELIMINARES DEL HSCL-A ADAPTADO PARA ADOLESCENTES URUGUAYOS EN CONTEXTO DE VIOLENCIA
}

\section{PRELIMINARY PSYCHOMETRIC CHARACTERISTICS OF THE HSCL-A ADAPTED FOR USE IN URUGUAYAN ADOLESCENTS IN THE CONTEXT OF VIOLENCE}

\author{
Cindy Mels \\ Universidad Católica del Uruguay \\ Daniel Trías \\ Universidad Católica del Uruguay
}

\begin{abstract}
Resumen: Este estudio tiene como objetivo realizar la adaptación lingüística y validación del Hopkins Symptom Checklist para Adolescentes (Bean et al., 2007), un instrumento de autoinforme breve, ampliamente usado en la detección de problemas internalizantes y externalizantes en adolescentes expuestos a situaciones de violencia. Tras la adaptación ligüística, involucrando a profesionales y adolescentes uruguayos, se administró el instrumento en una muestra de conveniencia compuesta por 529 adolescentes entre 12 y 16 años, alumnos de tres colegios privados católicos montevideanos de nivel socioeconómico medio-alto $(46,1 \%)$ y medio-bajo (53,9\%). También se aplicaron el IES-R (Weiss \& Marmar, 1997), BIEPS-J (Casullo, 2002) y cuestionario sociodemográfico. Los resultados del análisis de componentes principales respaldan al modelo de dos factores establecido en estudios previos. Se encontraron valores de Alpha de Chronbach satisfactorios (.90 para la escala total; .90 y .75 para las subescalas) comparables con los establecidos anteriormente. Se confirma la correlación positiva del HSCL-A con síntomas de estrés postraumático, su relación inversa con bienestar psicológico, y su capacidad para diferenciar entre víctimas y testigos de violencia, apoyando la validez convergente del instrumento. Concluimos el HSCL-A puede ser considerado como un instrumento sólido y prometedor para la evaluación de problemas internalizantes y externalizantes en adolescentes uruguayos.
\end{abstract}

Palabras Clave: violencia, desarrollo psicológico, problemas internalizantes, problemas de conducta, adolescencia

\begin{abstract}
The purpose of this study is the linguistic adaptation and validation of the Hopkins Symptom Checklist for adolescents (Bean et al., 2007), a self-administered brief screening instrument, widely used for the detection of internalizing and externalizing problems. Following its linguistic adaptation - involving Uruguayan professionals and adolescents - the instrument was administered in a convenience sample of 529 adolescents aged 12 to 16 years, students of three catholic private secondary schools in Montevideo, of medium high $(46,1 \%)$ and medium low $(53,9 \%)$ socioeconomic level. We also administered the IES-R (Weiss \& Marmar, 1997), BIEPS-J (Casullo, 2002) and a sociodemographic questionnaire. The results of the principal component analysis support the two factor model established in previous studies. Chronbach alpha values were satisfactory (.90 for the total scale and .90 and .75 for the subscales) comparable to those established in previous studies. Analysis confirmed the positive correlation between the HSCL-A scores and posttraumatic stress symptoms, its inversed correlation with psychological wellbeing, and its capacity to distinguish between victims and witnesses of violence, supporting convergent validity of the instrument. We conclude that the HSCL-A may be considered as a solid and promising instrument for evaluation internalizing and externalizing problems in Uruguayan adolescents.
\end{abstract}

Keywords: violence, psychological development, internalizing problems, behavior problems, adolescence

\section{Introducción}

La exposición a la violencia - tanto el ser testigo de actos violentos como la victimización directa - en niños y adolescentes ha sido relacionada con problemas de salud (Aber, Bennett, Conley \& Li, 1997), problemas emocionales y conductuales (Mels, 2012), así como, el fracaso escolar (Rosenfeld, Richman, Bowen \& Wynns, 2006). Inicialmente la investigación acerca de las consecuencias psicológicas de la exposición a la violencia, se ha enfocado en situaciones de abuso sexual, desastres naturales y la guerra (Horowitz, McKay \& Marchall, 2005; Mels, Derluyn, Broekaert \& Vlassenroot, 2012). En las últimas décadas surgió el interés por conocer el impacto de la violencia que se presenta en ámbitos urbanos sobre el desarrollo de niños y adolescentes (Henrich, Schwab-Stone, Fanti, Jones \& Ruchin, 2004; Steinbrenner, 2010; Horowitz et al., 2005).

Cada vez más se reconoce que la violencia forma parte de la vida cotidiana de niños y jóve-

Correspondencia: Cindy Mels.Facultad de Psicología. Universidad Católica del Uruguay.

Correo Electrónico: cimels@ucu.edu.uy 
nes en las grandes ciudades. Allí se perciben crecientes niveles de violencia y crímenes variados en el espacio público (acoso, rapiñas, uso de armas, presencia de pandillas y drogas, etc.). Este fenómeno es denominado violencia comunitaria (Lynch, 2003). La adolescencia puede considerarse como una etapa del ciclo vital con un elevado riesgo para la exposición a la violencia comunitaria, dado el mayor grado de independencia y autonomía que se establecen en la transición de la infancia a la adultez, implicando el aumento de interacciones potencialmente adversas fuera del hogar (Aisenberg \& Herrenkohl, 2008; Bowen \& Bowen, 1999; Rosenfeld et al., 2006).

Respecto a las consecuencias de la exposición a la violencia comunitaria en el desarrollo socio-afectivo de niños y adolescentes, el nivel internacional se suele usar el modelo internalizante - externalizante para la conceptualización de problemas emocionales y conductuales en estos grupos etarios. La dimensión externalizante abarca comportamientos disruptivos, tal como peleas, desobediencia, rabia, destrucción, agresividad física o verbal, y conductas delictivas, mientras que la internalizante hace referencia a estados de tensión psicológica en el propio sujeto, manifestados a través de conductas depresivas, ansiosas, el retraimiento, quejas somáticas y problemas de relacionamiento interpersonal (Lemos, Vallejo, \& Sandoval, 2002). Esta taxonomía ha sido basada en evidencias empíricas (Achenbach \& Edelbrock, 1978; Daset, Lopez-Soler, \& Hidalgo, 2009) y es considerada como un marco particularmente útil para la evaluación de psicopatología en comorbididad con estrés postraumático (Bean et al., 2007), típica en población afectada por la violencia.

Gran parte de los trabajos de investigación sobre las consecuencias psicologías de la exposición a la violencia comunitaria en adolescentes, han observado síntomas relacionados con el Trastorno de Estrés Postraumático (TEPT), siendo altas las tasas en adolescentes víctimas y testigos de violencia (Horowitz et al., 2005; Lynch, 2003). Por otro lado, se ha destacado la alta prevalencia de problemas externalizantes en adolescentes viviendo en comunidades con altas tasas de violencia, tal como conductas agresivas y delincuencia (Copeland-Linder, Lambert, \& lalongo, 2010; Gorman-Smith \& Tolan, 1998; Lambert, lalongo, Boyd, \& Cooley, 2005; Ludwig \& Warren, 2009; Osypuk et al., 2012).

Aunque menos investigado, en los últimos años varios estudios han vinculado la expo- sición violenta a la incidencia de problemas internalizantes, como la depresión y la ansiedad (Copeland-Linder et al., 2010; Grant et al., 2005; Ludwig \& Warren, 2009; Salzinger, Feldman, Stockhammer, \& Hood, 2002). Si bien es necesario seguir sumando estudios sobre esta relación en distintas comunidades, algunos autores han conectado este cuadro específico de síntomas con la exposición continua y prolongada a la violencia comunitaria (Cooley-Strickland et al., 2009; Fowler, Tompsett, Braciszewski, Jacques-Tiura, \& Baltes, 2009) y la coincidencia con otras fuentes de estrés, como problemas económicos (Mels, Derluyn, Broekaert, \& Rosseel, 2010a; Salzinger et al., 2002).

La variedad de síntomas asociados a la exposición a actos violentos demuestra que no todos los adolescentes responden de la misma manera (Gorman-Smith, \& Tolan, 1998; Lynch, 2003). Un gran desafío para la investigación actual y futura es ampliar el entendimiento de cómo se produce esa diversidad, especialmente, las respuestas resilientes observadas en un segmento considerable de la población expuesta a actos violentos (Gorman-Smith, \& Tolan, 1998).

\section{Variables moderadoras: exposición a la violencia, características personales y los contextos socioeconómicos}

Existe evidencia acumulada para ciertas variables que operan como moderadoras entre la exposición a la violencia y sus efectos psicológicos. En el marco de este trabajo estas relaciones establecidas dan insumos para el estudio de la validez convergente del instrumento a prueba. Una primera variable considerada es la acumulación de eventos violentos experimentados, que está positivamente relacionada con la cantidad y severidad de problemas psicológicos reportados (Lambert et al., 2005; Mels, 2012). Además, algunos estudios revelaron un efecto diferencial según dos grandes tipos de exposición: el ser testigo y el ser víctima directa del evento violento. En general, las víctimas suelen experimentar más problemas psicológicos que los testigos de actos violentos (Gorman-Smith \& Tolan, 1998; Kliewer, Lepore, Oskin, \& Johnson, 1998). Sin embargo, algunos estudios han mostrado que los dos tipos de exposición generan reacciones psicológicas diferentes. La victimización ha sido relacionada principalmente con problemas internalizantes, mientras que el ser testigo de eventos violentos muestra 
mayor correlación con problemas externalizantes (Fowler et al., 2009; Lynch, 2003). Siguen siendo poco claras las razones de esta relación. Se ha buscado la explicación en efectos neurofisiológicos diferenciales de la victimización frente al ser testigo de actos violentos (Henrich et al., 2004). Las teorías de aprendizaje social han conceptualizado la mayor prevalencia de problemas conductuales tanto como causa y efecto de la mayor frecuencia de presenciar actos violentos (Lynch, 2003).

Una segunda variable considerada como moderadora está constituida por algunas características individuales (sexo, edad) de las víctimas y los testigos de violencia en el ámbito urbano que han sido asociadas con diferentes respuestas psicológicas. En cuanto a la variable sexo, las evidencias coinciden en que los varones sufren generalmente niveles más altos de exposición a actos violentos, ya que suelen contar con menor supervisión parental y mayor frecuencia de interacciones problemáticas con pares, comparados con chicas (Cooley-Strickland et al., 2009; Osypuk, 2012). No obstante, respecto a los efectos psicológicos de la exposición violenta según sexo, los resultados son menos consistentes. Algunos estudios informan sobre la mayor prevalencia de problemas inter y externalizantes en las mujeres frente a varones (Gorman-Smith \& Tolan, 1998; Lynch, 2003). Otros autores sugieren que el impacto de la variable sexo difiere en función de la edad y el contexto específico investigado (Cooley-Strickland et al., 2009; Grant et al., 2005; Osypuk, 2012). Siguiendo con la influencia de la edad, existe una clara relación lineal positiva entre la edad y la cantidad de eventos violentos experimentados, tanto en términos de prevalencia por año como a lo largo de la vida (Fowler et al., 2009; Lambert et al., 2005). La edad también influye en la vulnerabilidad de adolescentes a las consecuencias psicológicas negativas de la exposición violenta (Grant et al., 2005). Un meta-análisis de la literatura concluyó que menor edad está asociada con mayor prevalencia de síntomas internalizantes, mientras que los adolescentes mayores suelen demostrar más problemas externalizantes que sus pares más jóvenes (Fowler et al., 2009).

La tercera variable a considerar es el contexto socioeconómico ya que se reconoce que la exposición a actos violentos y sus efectos negativos son superiores en contextos desfavorecidos (Gustafsson, Larsson, Nelson, \& Gus- tafsson, 2009). Esta elevada vulnerabilidad ha sido explicada por el estrés acumulativo generado por problemas económicos y la exclusión social característicos de una vida en pobreza (Gustafsson et al., 2009; Mels et al., 2010a; Miller \& Rasmussen, 2010; Seiffge-Krenke, 2000).

\section{Violencia Comunitaria y salud mental}

A pesar de los avances hechos en la última década, las evidencias sobre la violencia comunitaria y sus efectos en la salud mental presentan varias limitaciones. Gran parte de la investigación sobre el tema ha sido llevada a cabo en los Estados Unidos, haciendo necesario una mayor diversidad en muestras investigadas (Cooley-Strickland et al., 2009). Por otro lado, la dependencia de estudios transversales impide llegar a datos concluyentes respecto a los efectos de la exposición a actos violentos y el papel mediador de ciertas variables (Fowler et al., 2009; Gorman-Smith \& Tolan, 1998). Además, el uso de instrumentos sin respaldo psicométrico, debilita el estado del arte (CooleyStrickland et al., 2009). Para que se pueda dar paso a la elaboración de intervenciones preventivas, se necesitan estudios longitudinales que incluyan a adolescentes expuestos a variados tipos y niveles de violencia, que busquen mejorar el entendimiento de sus efectos en el desarrollo, y el rol mediador de factores de riesgo y protección (Aisenberg \& Herrenkohl, 2008; Cooley-Strickland et al., 2009).

Reconocido por la Organización Mundial de la Salud ([OMS], 2002) como un problema para la salud pública a escala mundial - particularmente en comunidades caracterizadas por grandes desigualdades económicas y sociales - el aumento de la violencia comunitaria plantea nuevos desafíos para familias, profesionales y diseñadores de políticas (Aisenberg \& Herrenkohl, 2008; Steinbrenner, 2010). En los últimos años, el problema se hizo particularmente visible en América Latina, la región con las tasas más altas a nivel mundial de homicidios, asaltos y robos - entre otros - en áreas metropolitanas (Moser \& Mcilwaine, 2006). En Uruguay se constata una evolución similar. En su análisis de tasas de delitos entre 1985 y 2007 en el país, Paternain (2008) concluyó que aumentó la frecuencia de delitos contra personas, homicidios, lesiones, denuncias contra la propiedad, delitos sexuales, rapiñas y daños, siendo el porcentaje más alto en zonas 
con desempleo alto y pobreza intensificada. Por otro lado, Munyo (2012) señala que esta evolución afecta particularmente a la población de adolescentes: están cada vez más involucrados como autores tanto como víctimas de violencia.

\section{Enunciado del problema y objetivos del estudio}

Se observa que la exposición a la violencia comunitaria afecta de modo creciente a los adolescentes en Uruguay. Por tanto, es relevante generar conocimiento científico sobre el alcance del problema y los procesos a través de los cuales su desarrollo psicológico resulta afectado. Para facilitar la investigación sobre la exposición violenta y sus consecuencias para la salud mental en adolescentes uruguayos, es necesario poder contar con instrumentos de investigación que cumplan con requisitos metodológicos actuales (Muñiz, Elosua \& Hambleton, 2013).

La evaluación psicológica eficaz de adolescentes en situación de violencia u otros siniestros, según Balaban (2006) tendría que ser breve, adaptada culturalmente y lingüísticamente a la población objetivo, y que preferentemente obedeciera al modelo de auto informe. Daset y sus colegas han trabajado en la adaptación, el desarrollo y la validación de instrumentos de tipo autoinforme para la detección de problemas inter y externalizantes en adolescentes uruguayos. En sus trabajos han utilizado el Youth Self Report (Daset et al., 2009) y el Autoinforme de Adolescentes -ADA- (Daset, López, Fernández, Benedetti, \& Costa Ball, 2013; Daset, Costa Ball, \& Fernández, 2014), pero hasta el momento no se ha llegado a la terminación de una lista abreviada de síntomas basada en evidencias en nuestro medio.

Frente a esta realidad, optamos por adaptar el Hopkins Symptoms Checklist for Adolescents ([HSCL-A], Bean, Derluyn, Eurelings-Bontekoe, Broekaert \& Spinhoven, 2007), a la población adolescente de Uruguay, e iniciar el proceso de su validación en nuestro medio. Este instrumento de tipo screening cumple con los requisitos que se plantean para una evaluación psicológica eficaz en esta temática. Su utilización internacional y sus propiedades psicométricas ya analizadas ofrecen una base amplia para la comparación futura de los datos sobre adolescentes uruguayos con otras poblaciones internacionales.

Los objetivos específicos del estudio son los siguientes:
- Adaptación lingüística del instrumento para uso en adolescentes (12 a 16 años) en Uruguay, a través de la revisión por profesionales y adolescentes.

- Evaluación y adaptación del procedimiento para la aplicación del instrumento en el medio escolar, en base de un estudio piloto.

- Evaluación preliminar de las características psicométricas del instrumento en una muestra de conveniencia de 3 colegios privados en Montevideo, de variado nivel socio-económico.

En concordancia con los autores del HSCL-A (Bean et al., 2007), la evaluación psicométrica existe del estudio de su consistencia interna, la exploración de su estructura factorial y la comprobación de sus relaciones teóricas persistentes en la literatura como indicador para la validez convergente del instrumento. Las hipótesis son las siguientes:

- Los adolescentes que se identifiquen como testigos de violencia tendrán puntuaciones más altos en la escala externalizante del HSCL-A frente a los que no testiguaron actos violentos (Lynch, 2003; Fowler et al., 2009).

- Los adolescentes que se identifiquen como víctimas de violencia tendrán puntuaciones más altas en ambas subescalas del HSCL-A frente a los que no se hicieron víctima de violencia (Gorman-Smith \& Tolan, 1998; Kliewer et al., 1998).

- Se espera una correlación fuerte y positiva entre la subescala de problemas internalizantes del HSCL-A y el Impact of Event Scale-Revised (Weiss \& Marmar, 1997), dado a la alta comorbididad entre síntomas de TEPT y problemas internalizantes (Bean et al., 2007).

- De acuerdo con teorías salugénicas que vean a la ausencia de síntomas de psicopatología como un aspecto del bienestar psicológico, se asume una correlación inversa entre la cantidad de síntomas de psicopatología (puntaje total HSCL-A) y el bienestar psicológico (Keyes, 2005).

\section{Metodología}

\section{Instrumentos iniciales}

- El HSCL-A (Bean et al., 2007), mide problemas internalizantes: síntomas basados en los criterios del DSM-IV depresión (14 ítems) y ansiedad (10 ítems) - y problemas externalizantes ( 7 ítems, correspondiendo a los criterios para el trastorno disocial y trastorno negativista desfiante según el DSM-IV, y 5 ítems relacionados 
al abuso de sustancias). El instrumento es una modificación del HSCL-25 (Derogatis, Lipman, Rikels, Ulenhoth, \& Colvi, 1974), desarrollado específicamente para adolescentes proviniendo de varias culturas (Bean et al., 2007). La escala está disponible en 19 idiomas - entre ellos el Español - con valores adecuados de validez de constructo, contenido y criterio, obtenido en una muestra de adolescentes inmigrantes y autóctonos holandeses y belgas (Bean et al., 2007), y adolescentes afectados por la guerra en Congo (Mels, Derluyn, Broekaert, \& Rosseel, 2010b). En ambos estudios se confirmó la estructura factorial basada en la dicotomía internalizante-externalizante.

En la versión original (Bean et al., 2007), 37 ítems adaptados a la vida adolescente evalúan la severidad de los síntomas en una escala Likert de 1 hasta 4 (nunca $=1$, a veces $=2$, seguido $=3$, siempre $=4$ ). Por esta cantidad de ítems, el instrumento fue dominado HSCL-37A por Bean y sus colegas (2007). No obstante, estudios psicométricos han mostrado que el ítem "no tener interés sexual" tiene un valor discriminatorio dudable. Por consiguiente, se ha sugerido quitar este ítem, reduciendo la lista hasta 36 ítems (Bean et al., 2007; Mels et al., 2010b). Para evitar confusión, y para aclarar que se trata de la versión de 36 ítems usamos la denominación HSCL-A a través de este trabajo.

Incluimos dos instrumentos para poder investigar la validez convergente del HSCL-A: El Impact of Event Scale-Revised (Escala Revisada del Impacto del Estresor; IES-R) (Weiss \& Marmar, 1997) (actualmente en proceso de validación para uso en adolescentes uruguayos) y la Escala de Bienestar Psicológico para adolescentes (BIEPS-J) (Casullo, 2002).

- El IES-R (Weiss \& Marmar, 1997), calcula los síntomas de estrés pos-traumático según los criterios del DSM-IV, a través de 22 ítems evaluando tres clúster de síntomas: intromisión (7 ítems), evitación (8) y excitación (7). Los ítems indagan la cantidad de dificultades que los encuestados han experimentado en la semana pasada en una escala de 0 (nunca), 1 (un poco), 2 (moderadamente), 3 (con frecuencia), 4 (extremamente). Aunque que esta escala fue desarrollada para el uso en poblaciones de adultos, se demostró apropiada para la evaluación de adolescentes à través de varios estudios internacionales (Balaban, 2006; Mels et al., 2010b). Para nuestro estudio partimos de la versión española (Baguena et al., 2001) que ha mostrado propiedades psicométricas aceptables en una muestra compuesta por 1078 adultos jóvenes.

- El BIEPS-J es una adaptación de la Escala de Bienestar Psicológico de Ryff para la población adolescente argentina, por parte de Casullo (2002). La escala autoadministrable mide cuatro de las seis dimensiones de bienestar psicológico propuestas por Ryff (1989): autonomía, control de situaciones, vínculos psicosociales y aceptación de sí mismo. Para la aplicación, los sujetos deben leer cada uno de los 13 ítems y responder qué sintieron y pensaron durante el último mes, eligiendo entre tres opciones de respuesta: de acuerdo (3 puntos); ni de acuerdo ni en desacuerdo (2 puntos); en desacuerdo (1 punto). La escala tiene confiabilidad aceptable (.70) en la población de adolescentes argentinos (Casullo, 2002).

- Por último, se incluyó un cuestionario socio-demográfico, evaluando a través de preguntas cerradas las variables edad, sexo, año cursando, el haber sido víctima de violencia y el haber sido testigo de violencia.

\section{Proceso de adaptación de los instrumentos para uso en adolescentes uruguayos}

Las 3 escalas fueron presentadas a una muestra de conveniencia de profesionales (3 académicos con experiencia clínica con adolescentes, 2 directores de colegios privados, 2 integrantes de una organización no-gubernamental que atiende a adolescentes), juntos con las definiciones de los conceptos medidos según el DSM-IV. Les pedimos que revisaran cada pregunta de los cuestionarios en relación al lenguaje empleado, teniendo en cuenta su elegibilidad para los destinatarios (adolescentes de variado nivel socio-económico) y la descripción conceptual de los constructos investigados. Para los ítems que señalaron como débiles o confusos, también pedimos sus sugerencias de cambio. Consiguientemente, los cuestionarios adaptados según las sugerencias de los profesionales fueron presentados individualmente a 4 adolescentes de variado nivel socio-económico (muestra de conveniencia). Les pedimos leer los ítems de los cuestionarios uno por uno y explicarnos a que se refería, verificando así su entendimiento. Para los ítems con mayores dificultades les pedimos sugerencias, llevando a otros refinamientos en la redacción de los ítems. 
Por último ejecutamos un estudio piloto en un grupo de 20 adolescentes de variado nivel socioeconómico en un colegio privado, para evaluar el procedimiento de aplicación de los cuestionarios.

\section{Estudio psicométrico: participantes y procedimiento}

Invitamos a tres colegios católicos privados - seleccionados por conveniencia, barrio y nivel socioeconómico - para participar en el estudio, a través de una carta explicando el diseño y objetivo del estudio y consecuentemente una reunión en sitio. Elegimos 3 barrios diferentes (Punta Carretas, Union y Maroñas) para asegurar mayor varianza en la muestra. Asimismo, dos de los colegios fueron clasificados según Beltrami (2004) en el estrato socioeconómico medio-bajo, uno en el estrato medio-alto.

Tras la aceptación institucional repartimos consentimientos informados para los padres de los alumnos del primer ciclo. Una semana después, se realizó la encuesta en el salón de clase, durante el horario escolar, sin la presencia del profesor. Se explicó el objetivo del estudio, una estimación de la duración de la actividad, el modo de contestar y las condiciones de la participación (voluntaria, anónima) antes del inicio de la encuesta. Durante esta introducción se destacó la incomodidad que podría generar la pregunta sobre los acontecimientos violentos, y los derechos de los participantes para no contestar o retirarse en cualquier momento de la encuesta. El proyecto y su procedimiento fueron aprobados por el comité de ética de la Facultad de Psicología de la UCU.

La Tabla 1 resume las características de la muestra recogida.

\section{Análisis}

Previo a los análisis se investigó la distribución de la variable dependiente en la muestra (prueba de Shapiro-Wilk, investigación del kurtosis/asimetría) y la adecuación de la muestra para análisis factorial (a través de la Medida de Adecuacion Muestral (KMO) y la prueba de esfericidad de Bartlett). El valor de KMO (.88) quedó adecuadamente encima de los .50 y la prueba de Bartlett fue significativa $\left(X^{2}=4809.84\right.$; $p=.000$ ) cumpliendo los criterios para análisis factorial. Aunque la muestra no mostró una distribución normal, no debilita el análisis factorial (Muthén \& Kaplan, 1985).
Tabla 1. Características de la muestra

\begin{tabular}{|c|c|c|c|}
\hline & & $\mathbf{N}$ & $\%$ \\
\hline \multirow[t]{3}{*}{ Sexo } & Varón & 267 & 50,5 \\
\hline & Mujer & 239 & 45,5 \\
\hline & Sin datos & 23 & 4,3 \\
\hline \multirow[t]{2}{*}{ Nivel socio-económico } & Medio-alto & 244 & 46,1 \\
\hline & Medio-bajo & 285 & 53,9 \\
\hline \multirow[t]{3}{*}{ Ha sido testigo de violencia } & Si & 279 & 52,7 \\
\hline & No & 237 & 44,8 \\
\hline & Sin datos & 13 & 2,5 \\
\hline \multirow[t]{3}{*}{ Ha sido víctima de violencia } & $\mathrm{Si}$ & 196 & 37,1 \\
\hline & No & 315 & 59,5 \\
\hline & Sin datos & 18 & 3,4 \\
\hline \multirow[t]{4}{*}{ Curso } & 1r año & 176 & 33,3 \\
\hline & $2^{\circ}$ año & 208 & 39,3 \\
\hline & $3 r$ año & 135 & 25,5 \\
\hline & Rango & Media & DT \\
\hline Edad & $12-16$ & 13,51 & ,98 \\
\hline HSCL total & $1,00-2,81$ & 1,58 & ,32 \\
\hline HSCL internalizante & $1,00-2,83$ & 1,68 &, 40 \\
\hline HSCL externalizante & $1,00-3,30$ & 1,35 & ,29 \\
\hline BIEPS & $13,00-39,00$ & 33,90 & 4,44 \\
\hline IES-R & $0,00-3,64$ & 1,09 &, 73 \\
\hline
\end{tabular}

La consistencia interna de la escala total y sus dos subescalas fue evaluada calculando el Cronbach's Alpha y la correlación de las subescalas. Ejecutamos un análisis de componentes principales (análisis factorial exploratorio, AFE) con rotación del tipo "obelique" (promax) como indicador de la validez de constructo. Se usó .40 como punto de corte para las cargas factoriales, un poco más estricto que el umbral generalmente usado de .30 (Costello y Osborne, 2005). Para determinar el número de factores a extraer nos basamos en el gráfico de sedimentación, planteado por Costello y Osborne (2005) como el criterio empíricamente más acertado para esta tarea. Para facilitar la comparación con otros estudios realizados sobre la estructura factorial del HSCL-A, también se forzó la extracción de un modelo de dos factores.

En concordancia con los autores originales del HSCL-A y Mels et al. (2010b) investigamos asociaciones teóricas como indicadores de la validez convergente, a través de pruebas $U$ de Mann-Whitney para la comparación de grupos independientes, formados en función de una variable categórica binaria (hipótesis 1 y 2) y correlaciones de Spearman (hipótesis 3 y 4). 


\section{Resultados}

\section{Estructura factorial}

Una primera AFE retuvo nueve factores con eigenvalores mayores a 1.00. No obstante, el gráfico de sedimentación mostró cinco factores antes del punto de inflexión, por lo cual se repitió el AFE forzando la extracción de cinco factores. En la Tabla 2 se presentan las cargas factoriales de los ítems en el modelo de cinco factores, explicando $45.79 \%$ de la varianza en la muestra. Los factores uno y dos abarcan todos los ítems de la escala internalizante del HSCL, sin diferenciar bien entre los ítems teóricamente propuestos como pertenecientes a la sintomatología de depresión por un lado, y ansiedad por otro. Se destacan los ítems 30 y 12, los únicos ítems que a la vez también tienen una carga considerable en los factores 3 y 4 respectivamente. Los ítems originalmente perteneciendo a la escala externalizante cargan todos en los

Tabla 2. Resultados análisis factorial exploratorio

\begin{tabular}{|c|c|c|c|c|c|c|c|}
\hline \multirow[b]{2}{*}{ Eigenvalue } & \multicolumn{5}{|c|}{ Modelo de 5 factores } & \multicolumn{2}{|c|}{$\begin{array}{l}\text { Modelo de } 2 \\
\text { factores }\end{array}$} \\
\hline & 8,253 & 2,879 & 2,167 & 1,706 & 1,481 & 8,253 & 2,879 \\
\hline \multicolumn{8}{|l|}{ Ítems escala internalizante } \\
\hline \multicolumn{8}{|l|}{ Depresión } \\
\hline 6 Te culpas por lo que te ocurre & ,623* & ,343 & , 184 & ,241 &,- 080 &, $603^{*}$ & ,214 \\
\hline 10 Te pones a llorar con facilidad &, $401^{*}$ &, $670^{*}$ & ,136 & ,228 &,- 194 &, $567^{\star}$ &, 051 \\
\hline 14 Tienes poca energía &, $409^{*}$ & ,367 &,- 001 & ,011 & ,135 &, $429^{*}$ & 097 \\
\hline 16 Tienes pocas ganas de comer & ,320 &, $509^{*}$ &, 081 & ,220 & 033 &, $443^{*}$ & ,135 \\
\hline $\begin{array}{l}19 \text { Tienes problemas para dormirte o te despiertas mucho } \\
\text { durante la noche }\end{array}$ &, $427^{*}$ &, 386 &, 161 &, 069 & ,242 &, $450^{*}$ & ,271 \\
\hline 22 Te sientes sin esperanza por el futuro &, $641^{*}$ & ,153 & ,295 &, 062 &, 164 &, $516^{*}$ &, 371 \\
\hline 23 No tienes ganas de hacer cosas &, $524^{*}$ & ,216 & ,153 & ,318 & ,199 &, $485^{*}$ & ,360 \\
\hline 26 Te sientes triste & ,729* &, $553^{*}$ & ,225 & ,218 &,- 114 &, $755^{*}$ & ,208 \\
\hline 29 Te sientes solo/a &, $726^{*}$ & ,423 & ,274 & ,094 &,- 004 &, $687^{*}$ & ,267 \\
\hline 30 Piensas en quitarte la vida &, $546^{*}$ & ,351 &, $523^{*}$ &,- 118 & ,355 &, $493^{*}$ &, $511^{*}$ \\
\hline 32 Piensas demasiado en las cosas &, $603^{*}$ & ,243 & ,107 & ,240 & 105 &, $547^{*}$ & ,268 \\
\hline 34 Tienes el sentimiento de que todo es muy difícil &, $671^{*}$ & ,355 &, 173 & ,219 & ,003 & $637^{*}$ & ,249 \\
\hline 35 Tienes sentimientos de que no vales nada &, $768^{*}$ &, $407^{*}$ & ,239 & ,200 & 064 &, $721^{*}$ & ,324 \\
\hline \multicolumn{8}{|l|}{ Ansiedad } \\
\hline 1 Te asustas así porque sí & ,226 & ,649* &,- 019 & ,258 &,- 084 & $436^{*}$ &,- 004 \\
\hline 2 Te sientes intranquilo, no te puedes quedar quieto/a &, 368 &, $423^{*}$ &, 066 &, 513 &, 040 &, $478^{*}$ & ,243 \\
\hline 5 Te sientes angustiado/a &, $709^{*}$ &, $515^{*}$ & ,225 & ,303 &,- 186 &, $738^{*}$ & ,200 \\
\hline 9 Te mareas & ,288 &, $569^{*}$ &, 164 &, 053 &, 171 &, $419^{*}$ & 189 \\
\hline 12 Te sientes nervioso/a & ,389 &, $516^{*}$ &,- 055 & ,449* &,- 196 &, $528^{*}$ & ,020 \\
\hline 15 Sufres latidos fuertes de corazón &, $522^{*}$ &, $434^{*}$ & ,226 &, 075 & ,207 &, $537^{\star}$ & ,304 \\
\hline 18 Tiemblas &, $454^{*}$ &, $482^{*}$ &, 078 & ,162 & ,120 &, $520^{*}$ & ,182 \\
\hline 21 Te sientes tenso/a & ,685* & ,395 & ,173 & ,279 &,- 048 & ,670* & ,240 \\
\hline 25 Tienes dolor de cabeza & ,346 &, $591^{*}$ & ,125 &, 079 &, 080 &, $475^{*}$ & ,135 \\
\hline 28 Sufres ataques de miedo & ,253 &, $589^{*}$ &,- 031 &,- 010 & ,161 &, $397^{*}$ & 036 \\
\hline 31 Te sientes ahogado/a o encerrado &, $652^{*}$ & ,307 & ,286 &,- 042 & ,194 &, $569^{*}$ & ,331 \\
\hline \multicolumn{8}{|l|}{ Ítems escala externalizante } \\
\hline 3 Te enojas rápidamente & ,287 & ,378 & ,210 & $608^{*}$ &,- 032 & $413^{*}$ & ,318 \\
\hline 4 Tomas alcohol cuando sales el fin de semana & ,283 &, 067 & ,801* & ,192 &, 153 & ,234 &, $670^{*}$ \\
\hline 7 Amenazas a otros & ,211 & ,071 & ,240 & ,395 &, $590^{*}$ & ,203 & ,603* \\
\hline 8 Fumas cigarrillos & ,197 &, 096 &, $767^{*}$ &, 027 & ,243 & ,163 & ,623* \\
\hline 11 Rompes o destruyes cosas de otros & 021 &, 085 &, 177 &, 144 &, $619^{*}$ & ,044 &, $464^{*}$ \\
\hline 13 Empiezas las peleas & ,163 &, 092 &, 152 &, $685^{*}$ &, 269 & ,219 &, $472^{*}$ \\
\hline 17 Haces mal a otro a propósito & ,083 &,- 077 & ,070 & ,307 &, $578^{*}$ & ,049 & ,459* \\
\hline 20 Te peleas & ,255 &, 117 & ,182 &, $710^{*}$ &, 124 &, 300 &, $437^{*}$ \\
\hline 24 Tomas alcohol durante la semana & ,206 &, 113 &, $719^{*}$ &, 069 & ,300 &, 179 & ,636* \\
\hline 27 Tomas pastillas para dormir y/o calmantes & ,283 & ,072 & ,317 &,- 217 &, $436^{*}$ & ,190 & ,382 \\
\hline 33 Robas cosas & ,131 & ,202 &, 172 &,- 067 & ,611* &, 144 & ,393 \\
\hline 36 Usas drogas (marihuana, cocaína, pasta base, otros) & ,234 &,- 020 &, $705^{*}$ &, 119 & ,091 & ,161 &, $557^{*}$ \\
\hline$\%$ varianza & 22,926 & 7,996 & 6,020 & 4,740 & 4,115 & 22,926 & 7,996 \\
\hline$\%$ varianza acumulada & & & 45,796 & & & 30 , & \\
\hline
\end{tabular}


tres factores restantes. El factor 3 (comprendido por los ítems 4, 8, 24 y 36) abarca el uso de sustancias, el factor 4 (ítems $3,13,20$ ) comprende conductas desafiantes, y el factor 5 (ítems 7 , $11,17,27,33$ ) engloba conductas antisociales.

En el modelo de dos factores (Tabla 2) se observa la replicación de la estructura internalizante (factor 1) vs. externalizante (factor 2) con excepción de dos ítems: el ítem 30 carga de manera alta en los dos factores, el ítem 3 tiene mayor carga en el factor 1 , a pesar de pertenecer teóricamente a la escala externalizante.

\section{Consistencia interna}

Los valores de Chronbach alfa fueron adecuados para el total de la escala $(\alpha=.899)$ y la subescala internalizante $(\alpha=.897)$ y aceptable para la subescala externalizante $(\alpha=.748)$.

\section{Validez convergente}

La Tabla 3 resume los resultados de las pruebas de comparación entre testigos y no testigos por un lado y las victimas y no victimas por otro lado, en los puntajes en la escala total y las subescalas del HSCL-A. Consistente con nuestros hipótesis, los testigos de actos violentos demuestran un puntaje significativamente más alto en la escala externalizante, en comparación con los que no han testigado violencia (hipótesis 1). Las víctimas por otro lado, tienen un puntaje significativamente mayor a los que no se hicieron víctima de violencia, tanto en la escala total, como en las dos subescalas (hipótesis 2).

Tabla 3. Comparación no paramétrica por grupos

\begin{tabular}{|c|c|c|c|}
\hline & $\begin{array}{c}\text { HSCL -A } \\
\text { total }\end{array}$ & $\begin{array}{c}\mathrm{HSCL}-\mathrm{A} \\
\text { internalizante }\end{array}$ & $\begin{array}{c}\mathrm{HSCL}-\mathrm{A} \\
\text { externalizante }\end{array}$ \\
\hline \multicolumn{4}{|c|}{ Testigos ( $n=277$ ) vs. no testigos $(n=236)$} \\
\hline \multicolumn{4}{|l|}{ Media (DT) } \\
\hline -No testigos & $1,55(, 32)$ & $1,67(, 41)$ & $1,30(, 28)$ \\
\hline -Testigos & $1,59(, 33)$ & $1,69(, 40)$ & $1,39(, 31)$ \\
\hline $\begin{array}{l}\text { U de Mann- } \\
\text { Whitney }\end{array}$ & 29982,50 & 31679,00 & 26148,50 \\
\hline $\mathbf{Z}$ & $-1,538$ &,- 521 & $-3,857$ \\
\hline $\begin{array}{l}\text { Tamaño de } \\
\text { efecto } r\end{array}$ &, 07 & 02 &, 17 \\
\hline$p$ & 124 & ,603 &, 000 \\
\hline \multicolumn{4}{|c|}{ Victimas $(n=194)$ vs. no victimas $(n=313)$} \\
\hline \multicolumn{4}{|l|}{ Media (DT) } \\
\hline -No victimas & $1,50(, 28)$ & $1,59(, 35)$ & $1,31(, 27)$ \\
\hline -Victimas & $1,69(, 35)$ & $1,82(, 48)$ & $1,41(, 32)$ \\
\hline $\begin{array}{l}\text { U de Mann- } \\
\text { Whitney }\end{array}$ & 20232,00 & 20853,50 & 23493,50 \\
\hline $\mathbf{Z}$ & $-6,319$ & $-5,930$ & $-4,307$ \\
\hline $\begin{array}{l}\text { Tamaño de } \\
\text { efecto } r\end{array}$ & ,28 & ,26 & ,19 \\
\hline$p$ & ,000 & 000 &, 000 \\
\hline
\end{tabular}

En la Tabla 4 se presentan las coeficientes de correlación Spearman entre los varios instrumentos aplicados. En referencia a la hipótesis 3 , se encontró una correlación fuerte $(r=.662)$ y significativa al nivel de confianza $p<.01$ entre la subescala internalizante del HSCL-A y los puntajes obtenidos en el IES-R. En relación a la hipótesis 4 , se halló una relación inversa moderada $(r=-.416)$ entre el total del HSCL y el BIEPS-J, significativa al nivel $p<.01$.

Tabla 4. Coeficientes de correlación de Spearman

\begin{tabular}{lccc}
\hline & & & \\
\cline { 2 - 4 } HSCL-A & & & \\
\hline Internalizante & - & - & - \\
Externalizante &, $445^{* *}$ & - & - \\
\hline Total &, $958^{* *}$ &, $653^{* *}$ & - \\
\hline & & & \\
IES-R &, $662^{* *}$ &, $397^{* *}$ &, $669^{* *}$ \\
BIEPS-J &,$- 395^{* *}$ &,$- 327^{* *}$ &,$- 416^{* *}$ \\
\hline${ }^{* *} p<0.01$ & & &
\end{tabular}

\section{Discusión}

A la hora de necesitar instrumentos adaptados al contexto uruguayo para poder avanzar nuestros conocimientos sobre las consecuencias psicológicas de la exposición a la violencia en adolescentes, este estudio abre el camino hacia el uso de HSCL-A con tal objetivo. Tras un proceso de adaptación lingüística informada por profesionales y adolescentes y la evaluación del procedimiento para su aplicación por medio de un estudio piloto, este primer estudio psicométrico del instrumento (incluyendo 529 alumnos de tres colegios privados montevideanos), demuestra características satisfactorias y comparables con las establecidas anteriormente en otros países (Bean et al., 2007; Mels et al., 2010b).

Se destaca la concordancia del modelo de dos factores (problemas internalizantes vs. externalizantes) con los datos psicométricos obtenidos por los autores originales (Bean et al., 2007), particularmente en cuanto al valor de la varianza explicada por el modelo (33.4\% frente a $30.9 \%$ en nuestro estudio), la consistencia interna de la escala total (.90 frente a .90 en nuestra muestra) y de sus subescalas (internalizante: .92 frente a .90; externalizante: .75 frente a .75). En cuanto a la carga factorial de los ítems en este modelo bifactorial, resaltan 2 ítems que también resultaron como más débil en estudios anteriores. El ítem 3 ("Te enojas rápidamente") tiene mayor carga en el factor 
internalizante en la muestra, mientras que teóricamente pertenece al clúster de problemas externalizantes. No obstante, el mismo ítem resultó relativamente débil en algunas versiones lingüísticas en los estudios de Bean et al. (2007) y Mels et al. (2010b) por la misma razón. Vale mencionar que la irritabilidad y agitación, también están incluidos dentro de los criterios del DSM-IV para el diagnostico de un trastorno depresivo, explicando su relación con problemas internalizantes. El ítem 30 ("Piensas en quitarte la vida") tiene una carga mayor a .40 en ambos factores en la muestra. El mismo ítem tampoco logró distinguir bien entre el factor internalizante y el factor externalizante en el estudio de Mels et al. (2010b) con adolescentes congoleses. La agresión subyacente a este sentimiento podría explicar su vinculación con problemas externalizantes. Futuros estudios deberían prestar particular atención a estos ítems para evaluar su idoneidad.

En la solución factorial con cinco factores no se diferencian claramente los ítems de ansiedad de los de depresión, de acuerdo con la alta comorbilidad de ambos trastornos observada en otras poblaciones de víctimas de violencia (Bean et al., 2007; Ludwig \& Warren, 2009; Salzinger et al., 2002) y el concepto de problemas internalizantes propuesto por Achenbach y Edelbrock (1978). No obstante, en este modelo factorial los problemas externalizantes se agrupan según las suposiciones teóricas, siendo los criterios del DSM-IV para conductas desafiantes, los de conductas antisociales y otros ítems para el uso de sustancias. Notablemente, esta estructura no se ha reportado en estudios anteriores, a pesar de su relevancia teórica. Dicho agrupamiento podría explicar la inferior consistencia interna de la escala externalizante en este estudio y estudios previos (Bean et al., 2007; Mels et al., 2010b).

En cuanto a la validez convergente, investigada a través de la comprobación de sus relaciones teóricas persistentes en la literatura, se confirmaron las hipótesis. En concordancia con la literatura, los adolescentes testigos de violencia hallaron puntuaciones significativamente más altas en la escala externalizante del HSCL-A, comparado con los no-testigos (Lynch, 2003; Fowler et al., 2009) y las victimas obtuvieron puntuaciones más altas en toda la escala, frente a los que no fueron víctimas de violencia (Gorman-Smith \& Tolan, 1998; Kliewer et al., 1998). Este último dato parece confirmar que la victimización es una experiencia psicológicamente más perturbadora que la observación de actos violentos dirigidos hacia otros, afectando a todo el espectro de emociones y conductas (Gorman-Smith \& Tolan, 1998). Por otro lado, se destaca que la asociación entre la observación de violencia y la manifestación de problemas externalizantes no necesariamente es causal, tal como propone la teoría de aprendizaje social. Varios autores han propuesto que adolescentes con problemas de conducta, manifestándose a través de la desobediencia, abuso de sustancias y conductas delictivas, suelen encontrarse con más frecuencia en situaciones potencialmente violentas (Fowler et al., 2009; Lynch, 2003).

Se confirmó la comorbilidad entre síntomas de TEPT y problemas internalizantes, a través de una correlación de .66 entre la escala internalizante del HSCL-A y el IES-R, comparable con la encontrada anteriormente por Mels et al. (2010b) $(r=.64)$. Por último, la correlación inversa encontrada entre el HSCL-A y el BIEPS-J, confirma la relación entre problemas psicológicos reportados y un descenso en el bienestar psicológico (Keyes, 2005). Entonces, estos datos agregan evidencias sobre la solidez del instrumento en la población investigada.

No obstante, las limitaciones de este estudio deberían ser tomadas en consideración a la hora de interpretar y generalizar los resultados obtenidos. Entre ellas se destacan la naturaleza selectiva de la muestra, la técnica de análisis factorial usada (AFE en vez de análisis confirmatorio) y la no inclusión de procedimientos adicionales para la investigación avanzada de la fiabilidad y validez del instrumento (tal como, la inclusión de otros informantes, medición repetida, entrevista clínica). Recalcamos que el HSCL-A es una herramienta para la investigación y detección de problemas psicológicos, y no un instrumento diagnóstico. Para fortalecer esta función se recomienda que futuros estudios se dediquen a la obtención de datos normativos en una muestra representativa por un lado, y la evaluación de su validez de criterio frente a un estándar diagnostico establecido por otro lado.

No obstante, enfatizamos que el estudio psicométrico de instrumentos psicológicos es un proceso de larga duración y esfuerzo colectivo de acumulación de evidencias. Mostrando su potencial psicométrico, con este estudio esperemos ir abriendo camino hacia el uso del HSCL-A en la evaluación psicológica de adolescentes afectados por distintas formas de violencia en 
Uruguay, y la investigación avanzada de su validez y fiabilidad. A la vez los datos obtenidos van posibilitando dar pasos en la investigación de variables mediadores en la relación entre la exposición a la violencia y consecuencias emocionales, conductuales y académicas en adolescentes uruguayos.

\section{Referencias}

Aber, J., Bennett, D., Conley, D., \& Li, J. (1997). The effects of poverty on child health and development. Annual Review of Public Health, 18 (1), 463-483.

Achenbach, T., \& Edelbrock, C. (1978). The classification of child psychopathology: a review and analisys of empirical efforts. Psychological Bulletin, 85, 1275-1301.

Aisenberg, E. \& Herrenkohl, T. (2008). Community violence in context: risk and resilience in children and families. Journal of Interpersonal Violence, 23, 296-315.

Balaban, V. (2006). Psychological Assessment of Children in Disasters and Emergencies. Disasters, 30 (2), 178-198.

Baguena, M. J., Villarroya, E., Belena, A., Amelia, D., Roldan, C., \& Reig, R. (2001). Propiedades psicometricas de la version espanola de la Escala Revisada de Impacto del Estressor (EIE-R). Analisis y Modificacion de Conducta, 27, 581-604.

Bean, T., Derluyn, I., Eurelings-Bontekoe, E., Broekaert, E., \& Spinhoven, P. (2007). Validation of the multiple language versions of the Hopkins Symptom Checklist-37 for Refugee Adolescents. Adolescence, 42, 51-71.

Beltrami, M. (2004). Metodología aplicada para la estratificación de Montevideo Urbano y del Interior Urbano Montevideo: Instituto Nacional de Estadística.

Bowen, N., \& Bowen, G. (1999). Effects of crime and violence in neighborhoods and schools on the school behavior and performance of adolescents. Journal of Adolescent Research, 14 (3), 319-342.

Casullo, M. (2002). Evaluación del bienestar psicológico en Iberoamérica. Buenos Aires: Paidós.

Copeland-Linder, N., Lambert, S., \& lalongo, N. (2010). Community Violence, Protective Factors, and Adolescent Mental Health: A Profile Analysis. Journal of Clinical Child \& Adolescent Psychology, 39 (2).

Cooley-Strickland, M., Quille, T., Griffin, R., Stuart, E., Bradshaw, C., \& Furr-Holden, D. (2009). Community violence and youth: affect, behavior, substance use, and academics. Clinical Child and Family Psychological Review, 12, 127-156.

Costelo, A., \& Osborne, J. (2005). Best Practices in Exploratory Factor Analysis: Four Recommendations for Getting the Most From Your Analysis. Practical Assessment, Research \& Evaluation, 10 (7), 1-9.

Daset, L., Lopez-Soler, C., \& Hidalgo, M. (2009). Síndromes empíricos en una población adolescente, evaluados mediante el YSR. Ciencias Psicológicas, $3(1), 67-82$.

Daset, L.R., Costa Ball, D. \& Fernández, M.E. (2014). [Resultados de un estudio piloto con el Autoinforme de Adolescentes ADA]. Datos duros inéditos.

Daset, L., López, A., Fernández, M.E., Benedetti, M. \& Costa Ball, D. (Octubre, 2013). Estructura del Autoinforme de Adolescentes y resultados de la evaluación de jueces y jóvenes: consideraciones clínicas y psicométricas. Trabajo presentado en el IV Simposio de Investigación en Psicología. Universidad Católica del Uruguay.
Derogatis, L. R., Lipman, R., Rikels, K., Ulenhoth, E., \& Covi, L. (1974). The Hopkins Symptom Checklist (HSCL): A self-report symptom inventory. Behavioral Science, 19, 1-15.

Fowler, P., Tompsett, C., Braciszewski, J., Jacques-Tiura, A.J., \& Baltes, B. B. (2009). Community violence: a meta-analysis on the effect of exposure and mental health outcomes of children and adolescents. Development \& Psychopathol, 21, 227-259.

Gustafsson, P. E., Larsson, I., Nelson, N., \& Gustafsson, P. A.(2009). Sociocultural disadvantage, traumatic life events, and psychiatric symptoms in preadolescent children. American Journal of Orthopsychiatry, 79, 387-397.

Grant, K.E., McCormick, A., Poindexter, L., Simpkins, T., Janda, C.M., Thomas, K.J.,... Campbell A. (2005). Exposure to violence and parenting as mediators between poverty and psychological symptoms in urban African American adolescents. Journal of Adolescence, 28, 507-521.

Gorman-Smith, D., \& Tolan, P. (1998). The role of exposure to community violence and developmental problems among innercity youth. Development and Psychopathology, 10, 101-116.

Henrich, C., Schwab-Stone, M., Fanti, K., Jones, S., \& Ruchin, V. (2004). The association of community violence exposure with middle-school achievement: A prospective study. Applied Developmental Psychology, 25, 327-348.

Horowitz, , K., McKay, M., \& Marshall, R. (2005). Community violence and urban families: Experiences, effects, and directions for intervention. American Journal of Orthopsychiatry, 75, 356-368.

Keyes, C. (2005). Mental illness and/or mental health? Examinating axions of the Complete State Model of Health. Journal of Consulting and Clinical Psychology, 73 (3), 539-548.

Kliewer, W., Lepore, S., Oskin, D., \& Johnson, P. (1998). The Role of Social and Cognitive Processes in Children's Adjustment to Community Violence. Journal of Consulting and Clinical Psychology, 66 (1), 199-209.

Lambert, S.F., lalongo, N.S., Boyd, R.C., \& Cooley, M.R. (2005). Risk factors for community violence exposure on adolescents. American Journal of Community Psychology, 36, 29-48.

Lemos, S., Vallejo, G., \& Sandoval, M. (2002). Estructural factorial del Youth Self Report (YSR). Psicothema, 14 (4), 816-822.

Lynch, M. (2003). Consequences of children's exposure to community violence. Clinical Child and Family Psychology Review, 6 (4), 265-274.

Ludwig, K., \& Warren, J. (2009). Community violence, school-related protective factors, and psychosocial outcomes in urban youth. Psychology in the Schools, 46 (10), 1061-1073.

Mels, C. (2012). Entre Pobreza y violencia: ¿cómo afectan los estresores diarios y la violencia a adolescentes en zonas de guerra? Ciencias Psicologicas, 6 (2), 111-122.

Mels, C., Derluyn, I., Broekaert, E., \& Rosseel, Y. (2010a). The psychological impact of forced displacement and related risk factors on Eastern Congolese adolescents affected by war. Child Psychology \& Psychiatry, 51 (10), 1096-1104.

Mels, C., Derluyn, I., Broekaert, E., \& Rosseel, Y. (2010b). Community-based cross-cultural adaptation of mental health measures in emergency settings: validating the IES-R and HSCL-37A in Eastern Democratic Republic of Congo. Social Psychiatry \& Psychiatric Epidemiology, 45, 899-910. 
Mels, C., Derluyn, I., Broekaert, E., \& Vlassenroot, K. (2012). Exploring the context for adolescent mental health and psychosocial assistance in the Eastern Democratic Republic of Congo. En I. Derluyn, C. Mels, W. Vanhole \& P. Parmentier (Eds.). Re-member. Rehabilitation and Reintegration of War-Affected Children (pp. 475495). Leuven: Intersentia.

Miller, K., \& Rasmussen, A. (2010). War exposure, daily stressors; and mental health in conflict and postconflict settings: bridging the divide between traumafocussed and psychosocial frameworks. Social Science \& Medicine, 70, 7-16.

Moser, C., \& Mcilwaine, C. (2006). Latin American Urban Violence as a Development Concern: Towards a Framework for Violence Reduction. World Development, 34 (1), 89-112.

Munyo, I. (2012). Los dilemas de la delincuencia juvenil en Uruguay. Montevideo: Centro de Estudios de la Realidad Económica y Social (CERES).

Muñiz, J., Elosua, P., \& Hambleton, R. K. (2013). International Test Commission Guidelines for test translation and adaptation: Second edition. Psicothema, 25(2), 151-157.

Muthén, B., \& Kaplan D. (1985). A comparison of some methodologies for the factor analysis of non-normal Likert variables. British Journal of Mathematical and Statistical Psychology, 38, 171-189.

Organización Mundial de la Salud (2002). Reducir los riesgos y promover una vida sana. (Informe sobre la salud en el mundo 2002). Recuperado de http://www. who.int/whr/2002/es/

Osypuk, T., Schmidt, N., Bates, L., Tchetgen- Tchetgen , E., Earls, F., \& Glymour, M. (2012). Gender and crime victimization modify neighborhood effects on adolescent mental health. Pediatrics, 130 (3), 472-481.
Paternain, R. (2008). Los espacios regionales del delito en Uruguay. En R. Paternain \& R., Sansiviero (Eds.), Violencia, inseguridad y miedos en Uruguay ¿Qué tienen que decir las ciencias sociales? Montevideo: FESUR.

Rosenfeld, L., Richman, J., Bowen, G., \& Wynns, S. (2006). In the face of a dangerous community: the effects of social support and neighborhood danger on high school student's school outcomes. Southern Communication Journal, 71 (3), 273-289.

Ryff, C. (1989). Happiness is everything, or is it? Explorations on the meaning of psychological wellbeing. Journal of Personality and Social Psychology, 57, 1069-1081.

Salzinger, S., Feldman, R. S., Stockhammer,T., \& Hood, J. (2002). An ecological framework for understanding risk for exposure to community violence and the effects of exposure on children and adolescents. Aggression and Violent Behavior, 7, 423-45.

Seiffge-Krenke, I. (2000). Causal links between stressful events, coping style and adolescent symptomatology. Journal of Adolescence, 23, 675-691.

Steinbrenner, S. (2010). Concept analysis of community violence: using adolescent exposure to community violence as an exemplar. Issues in Mental Health Nursing, 31, 4-7.

Weiss, D., \& Marmar, C. (1997). The Impact of Event ScaleRevised. En T. Keane (Ed.), Assessing psychological trauma and PTSD (pp.168-189). New York: Guilford Press.

Para citar este artículo:

Mels, C., \& Trías, D. (2014). Características psicométricas preliminares del HSCL-A adaptado para adolescentes uruguayos en contexto de violencia. Ciencias Psicológicas VIII (2): 139 - 149. 